Published in final edited form as:

Evolution. 2020 November ; 74(11): 2451-2464. doi:10.1111/evo.14104.

\title{
Short-term heritable variation overwhelms two hundred generations of mutational variance for metabolic traits in Caenorhabditis elegans:
}

\section{Heritable Metabolic Variance}

\author{
Lindsay M. Johnson ${ }^{1,4}$, Olivia J. Smith ${ }^{1}$, Daniel A. Hahn ${ }^{2,3}$, Charles F. Baer ${ }^{1,3}$ \\ 1-Department of Biology, University of Florida, Gainesville, Florida, 32611 \\ 2 -Department of Entomology and Nematology, University of Florida, Gainesville, Florida, 32611 \\ 3 -University of Florida Genetics Institute, Gainesville, Florida, 32611 \\ 4-Present address: Ology Bioservices, Inc., Alachua, FL 32615
}

\begin{abstract}
Metabolic disorders have a large heritable component, and have increased markedly in human populations over the past few generations. Genome-wide association studies of metabolic traits typically find a substantial unexplained fraction of total heritability, suggesting an important role of spontaneous mutation. An alternative explanation is that epigenetic effects contribute significantly to the heritable variation. Here we report a study designed to quantify the cumulative effects of spontaneous mutation on adenosine metabolism in the nematode Caenorhabditis elegans, including both the activity and concentration of two metabolic enzymes and the standing pools of their associated metabolites. The only prior studies on the effects of mutation on metabolic enzyme activity, in Drosophila melanogaster, found that total enzyme activity presents a mutational target similar to that of morphological and life-history traits. However, those studies were not designed to account for short-term heritable effects. We find that the short-term heritable variance for most traits is of similar magnitude as the variance among MA lines. This result suggests that the potential heritable effects of epigenetic variation in metabolic disease warrant additional scrutiny.
\end{abstract}

\section{Keywords}

Epigenetic; Metabolic enzyme; Mutation accumulation; Mutational variance; transgenerational inheritance

\footnotetext{
Correspondence to: Charles F. Baer, Department of Biology/University of Florida Genetics Institute, P.O. Box 118525, University of Florida, Gainesville, FL 32611-8525 USA.

Author contributions: Conceived research: CFB; Designed experiments: LMJ, DAH, CFB; Conducted experiments: LMJ, OJS; analyzed data: LMJ, DAH, CFB; wrote manuscript: LMJ, DAH, CFB.

Data Accessibility Statement: Data are included in supplemental file "Supplemental Data" and are deposited in Dryad at https:// doi.org/10.5061/dryad.9cnp5hqgb.
} 


\section{INTRODUCTION}

Human metabolic diseases have increased markedly in frequency over the past few generations (Saklayen 2018). Large genome-wide association studies (GWAS) conducted on the human metabolome have shown that metabolic traits are highly heritable, but that a substantial fraction of the heritability of metabolic traits remains unexplained by the cumulative effects of mQTL (Rhee et al. 2013; Shin et al. 2014; Mahajan et al. 2018). This discrepancy indicates that the remainder of the heritable variation is the result of some combination of (1) rare, highly deleterious variants recently arisen in the population; (2) many variants with effects too small to be detected by the typical GWAS (Manolio et al. 2009; Eichler et al. 2010; Boyle et al. 2017); (3) epistasis (Zuk et al. 2012; Sackton and Hartl 2016), and/or (4) cross-generational epigenetic effects that are heritable over the short term but leave no genetic signature (Furrow et al. 2011; Richard et al. 2017). Scenarios (1) and (2) (and maybe 3) imply a significant role of spontaneous mutations at mutationselection balance in the risk of metabolic disease, although the rapid increase in frequency further implies some sort of genotype-environment interaction. A recent onslaught of epigenetic effects is considered less likely as a general explanation for the "missing heritability" of human complex traits (Wainschtein et al. 2019), but specific examples of cross-generational effects are known in humans (Pembrey et al. 2006; Curley et al. 2011; Veenendaal et al. 2013; Rando and Simmons 2015), and are well-documented in other organisms (e.g., plants; Munir et al. 2001; Luna et al. 2012; Rasmann et al. 2012) and $C$. elegans; (Greer et al. 2011; Rechavi et al. 2011; Ashe et al. 2012; Jobson et al. 2015; Marré et al. 2016).

To our knowledge, the cumulative effects of spontaneous mutation on metabolic traits have been investigated in only three experiments. Mukai et al. (1984) measured the cumulative effects of 300 generations of spontaneous mutations on the activity of alcohol dehydrogenase (Adh) in Drosophila melanogaster. In a groundbreaking study, also in Drosophila melanogaster, Clark et al. (1995) quantified the input of mutational (co)variance in the activity of a set of 12 metabolic enzymes and two metabolites. In both studies, mutational heritability $\left(h_{M}^{2}\right.$, the per-generation increase in genetic variation $\left(\mathrm{V}_{\mathrm{M}}\right)$ scaled as a fraction of the residual variance, $\mathrm{V}_{\mathrm{E}}$ ) of enzyme activity was on the order of that of lifehistory and morphological traits $\left(h_{M}^{2} \approx 10^{-3} /\right.$ generation; Houle et al. (1996)). In several of the mutation accumulation (MA) lines studied by Clark and his colleagues, there were large changes in enzymatic activity relative to the population mean over the course of 44 generations of evolution under minimal selection. Results for the two metabolites studied were analogous, but there was no attempt to assess the relationship between enzyme activity and metabolite concentration in the context of metabolic pathways.

More recently, Davies et al. (2016) examined the changes in metabolite concentration for 29 metabolites in a set of $C$. elegans MA lines that had undergone $\sim 250$ generations of evolution under minimal selection and found that metabolites vary considerably in their response to spontaneous mutation, as quantified by the change in mean metabolite concentration $(\Delta \mathrm{M})$ and by the mutational (co)variance. Associations between mutational correlations between pairs of metabolites ( $r_{M}$, presumably the result of pleiotropy) and 
proximity of the metabolites in the global metabolic network were, on average, positive but weak (Johnson et al. 2018). The weakness of the association between mutational pleiotropy and network proximity suggests that pleiotropic effects propagate throughout the metabolic network and are not confined to local modules. However, there was no attempt to link changes in metabolite concentration to the properties of associated metabolic enzymes.

Here we report results of a study designed to investigate the cumulative effects of mutation on the concentration and activity of the enzymes in the adenosine metabolism pathway and their associated metabolites (Figure 1), using (nearly) the same set of $C$. elegans MA lines as in Davies et al. (2016). We chose this particular metabolic pathway for investigation because adenosine was one of the metabolites with the largest mutational variance, indicative of a large mutational target. In addition, adenosine levels are assumed to be tightly regulated due to its role as a critical signaling molecule for energetic homeostasis as a metabolite involved in ATP: AMP, as well has having other critical functions (Park and Gupta 2008; Boison 2013). Lastly, the adenosine pathway has well-defined network topology and is highly conserved.

Our study has one additional important feature relative to the aforementioned ones (Clark et al. 1995; Davies et al. 2016; Johnson et al. 2018). All of those studies estimate cumulative mutational parameters from the among-line components of (co)variance of a set of MA lines. Ideally, the among-line (co)variance is due solely to the contribution of new mutations, but other technical and biological factors can contribute to the among-line (co)variance (Lynch and Walsh 1998). Our experimental design allows us to infer the relative contributions of both mutation and short-term heritable effects on the total heritable variance of metabolic traits.

\section{MATERIALS AND METHODS}

\section{Mutation Accumulation:}

A detailed description of the construction and propagation of the mutation accumulation (MA) lines is given in Baer et al. (2005). Briefly, 100 replicate MA lines were initiated from a nearly isogenic population of N2-strain $C$. elegans and propagated by singlehermaphrodite descent at four-day (one generation) intervals for approximately 250 generations. The common ancestor of the MA lines ("G0") was cryopreserved at the outset of the experiment; MA lines were cryopreserved upon completion of the MA phase of the experiment (Figure 2). Based on extensive whole-genome sequencing (Denver et al. 2012; Saxena et al. 2019), we estimate that the average MA line carries at least 60-100 mutant alleles in the homozygous state. In this study we included 39 of the 43 N2-strain MA lines assayed by Davies et al. (2016). One of the lines included in that study (line 507) was revealed by genome sequencing to be a contaminant from a different strain, and was removed from the analysis. Two other lines $(517,598)$ were revealed to have been crosscontaminated subsequent to the MA phase of the experiment, i.e., they appear to be genetically identical. Due to the structure of the experiment, we cannot simply pool the replicates of the two lines without introducing a potential bias, so those lines were omitted as well. All replicates of line 571 reproduced so slowly that we were unable to obtain sufficient material to be used in downstream assays. 
The ideal design of a phenotypic assay of a MA experiment includes replicates of the (putatively) unmutated common ancestor, which we call "pseudolines" and which are treated identically to MA lines in analyses (Lynch 1985; Lynch and Walsh 1998; Teotónio et al. 2017). The among-pseudoline component of variance includes the effects of residual segregating genetic variation in the ancestor, as well as short-term heritable effects that are propagated across assay generations and purely environmental effects resulting from (sometimes unavoidable) imperfections of experimental design, such as a temporal correlation between line and assay time. In the absence of a pseudoline control, some fraction of the among-MA line (co)variance will potentially be the result of non-mutational factors, and resulting estimates of $\mathrm{V}_{\mathrm{M}}$ and $\mathrm{COV}_{\mathrm{M}}$ will be upwardly biased.

Here, a set of 15 pseudolines (PS) of the G0 ancestor were included along with the MA lines (Figure 3A). PS lines were generated by thawing a sample of the N2 ancestor and allowing it 24 hours to recover from freezing, at which time 15 hermaphrodites were plated individually onto $60 \mathrm{~mm}$ NGM plates seeded with $100 \mu \mathrm{l}$ of an overnight culture of $E$. coli OP50 (P0 generation in Figure 3A). P0 worms were allowed to reproduce until the bacterial food on the plate was consumed (two generations; F1 and F2), at which time worms were cryopreserved (F2) (Hope 1999). The demographic features of this protocol mimic those of our standard protocol for cryopreserving MA lines. From this point forward, MA lines and ancestral PS lines are experimentally identical.

\section{Protein Extraction:}

This study includes six independent experimental tests: concentration and activity of two metabolic enzymes (ADA and ADK), total protein concentration, and mass spectrometry of pooled metabolites. We were unable to measure the activity of the third enzyme in the pathway, adenosine phosphoribosyltransferase (APRT), because commercially available assay kits require too much material to be practical for application to $C$. elegans.

Accordingly, six aliquots of protein (plus metabolites) were extracted and cryopreserved from the same individual sample of each experimental replicate. Protein extraction was performed in five blocks of 10-12 lines per block, to ensure that all samples were handled at the appropriate stage of development (see below). In each protein extraction block, the lines selected were a random mix of MA and PS lines; the experimental design is outlined in Figure 3B. Each line was thawed and transferred onto a $60 \mathrm{~mm}$ agar plate. The following day, five L4-stage hermaphrodites from each line were transferred individually onto $35 \mathrm{~mm}$ agar plates (parental generation, P1 in Figure 3B), resulting in a total of 290 samples (five replicates of each of 15 PS lines and 43 MA lines). Four days later, a single offspring (F1 generation) L4 hermaphrodite was transferred from each P1 plate onto a 100mm plate (F1.1 in Figure 3B). The F1 worms were grown for ten days (two generations, F2.1 and F3.1 in Figure 3B) of self-replication to ensure that F3 worms were gravid and there were abundant eggs on the plate (F4.1 in Figure 3B). Worms were washed from the plate and "bleached" in an $\mathrm{NaOH}$ and sodium hypochlorite solution (Sulston and Hodgkin 1988). This process kills all hatched worms by breaking down their cuticle and leaves only eggs (F4.1 in Figure 3B), resulting in a population that is closely synchronized in developmental timing. Once F4 worms had been bleached, hatched, and reached the L4 stage, they were washed five times in ion-free NGM buffer, mixed with protease inhibitor cocktail, and homogenized via 
sonication (Tang and Choe 2015). Homogenized samples were centrifuged, and the proteinrich supernatant was distributed equally into six cryovials and stored at $-80 \mathrm{C}^{\circ}$. All lines, both MA and PS, were labeled with their true line number until cryopreservation, at which time each replicate was assigned a random number to obscure sample identity.

\section{Estimating Total Soluble Protein via Bicinchoninic Acid Assay (BCA)}

We used total soluble protein as a proxy for the number of individual worms in a sample. To quantify the total soluble protein in each sample we used a bicinchoninic acid assay (BCA) following the protocol from Thermo Scientific (Pierce BCA Protein Assay Kit \#23225). Briefly, a set of known concentrations of bovine serum albumin is used to generate a standard curve against which one can estimate the concentrations of unknown protein samples. A total of $13 \mathrm{BC}$ assays were performed, each with its own set of standards.

\section{Enzyme activity assays:}

(i) Adenosine kinase (ADK) - Adenosine kinase (ADK) activity was measured using the Novocib PRECISE ADK assay kit (Novocib, Ref \#K0507-01). This assay measures $\mathrm{ADK}$ activity based on the production on $\mathrm{NADH}_{2}$, which is generated by the dephosphorylation of ATP by ADK. To ensure that ADK activity is not limited by available ATP, an excess of human ATP was added to each sample. Absorbance at 340nm was measured at one-minute intervals for 40 minutes. The slope of the line over the linear phase quantifies the activity of each sample in units of absorption per minute. A set of positive (human ADK, provided in the kit) and negative (no enzyme) controls were included with the unknown samples in each assay plate and used to quantify assay quality, per the manufacturer's instructions. ADK activity was assayed in five blocks. Thirty of the 290 samples were not included in the ADK activity assay because of erratic activity slopes. All samples that were run included at least two technical replicates, in which extracts from a sample were split and assayed independently.

(ii) Adenosine deaminase (ADA): ADA activity was measured using Abcam's Adenosine Deaminase (ADA) Activity Assay Kit (Abcam, ab21193). This kit utilizes an ADA developer and converter which react with inosine formed from the breakdown of adenosine by ADA to produce uric acid. Uric acid concentration is then measured via absorbance at $293 \mathrm{~nm}$ once a minute for 45 minutes. Each kit is run with a set of known concentration standards that are used to generate a standard curve. The quantity of uric acid was then measured and used to calculate the activity of the ADA in a given sample in units of $\mathrm{nmol} / \mathrm{min} / \mu \mathrm{g}$, following the manufacturer's instructions.

ADA activity was assayed in four 96-well plates, each including a positive (manufacturer supplied ADA) and negative (no sample) control. For one assay plate (= assay block), the highest concentration standard had an unusually low reading; we therefore omitted this point from the standard curve for this assay. Omission of that point had no effect on the interpretation of the data because all unknown samples had absorbance values greater than the second lowest standard. All of the 290 samples had maximum measured activity well below the highest concentration standard. Four samples with erratic absorption readings were omitted from further analyses. 


\section{Enzyme concentration:}

Enzyme concentrations were estimated by Western blot (WB) (Supplemental Figure S1). Extracted samples were denatured in 2X Laemmli buffer (with $\beta$-mercaptoethanol) and boiled at $70^{\circ}$ for 10 minutes. Each gel lane was loaded with $7 \mathrm{ug}$ of total soluble protein calculated from the BCA data (Bio-Rad 10\% polyacrylamide gel, product \#4561033). Each blot included eight samples, a DNA-ladder and an internal control standard consisting of a homogenate of $C$. elegans. We used the Trans-Blot Turbo Transfer System (Bio-Rad, \#1704156) to transfer proteins separated by gel electrophoresis onto blotting paper. After the primary (enzyme-specific) and secondary (visualization) antibodies were bound (antibodies described below), antibody binding was visualized using the Pierce ECL Western Blotting Substrate (Thermo Fischer Product \# 32106). Brightness of each band relative to the internal control was estimated using ImageJ image-analysis software and used as a proxy for enzyme concentration. 246 of the 284 samples contained sufficient protein to be visualized by Western Blot.

The concentration of tubulin in a sample is commonly used as a loading control, and we quantified tubulin in each sample for both enzymes (Tubulin antibody DSHB, E7). However, tubulin concentration was not independent of treatment (MA vs. PS), so we treat it as an experimental trait rather than a control (see Results).

(i) ADK concentration-The antibody used was Abcam's Anti-ADK antibody - Cterminal (Abcam, ab226187), which was designed and tested in mouse and humans and which is homologous with the $C$. elegans ADK protein, R07H5.8. The assay resulted in multiple binding sites, with distinct bands at $\sim 100 \mathrm{kd}, \sim 37 \mathrm{kd}, \sim 25 \mathrm{kd}$, and $\sim 18 \mathrm{kd}$ (Supplemental Figure S2). To determine which of these binding sites represented the $C$. elegans ADK, samples of each band were extracted from the gel and analyzed using protein mass spectroscopy. Results were then analyzed using Scaffold 4; only the sample at $\sim 37 \mathrm{kd}$ contained the worm ADK homolog (R07H5.8, molecular weight $=37.5 \mathrm{kd}$; Wormbase) 112 of the 246 samples did not contain sufficient ADK to be measured by Western blot. These lines were tested in duplicate and failed to produce ADK bands both times, therefore the low concentration of ADK is presumably a true property of the sample and not an experimental artifact.

(ii) Adenosine deaminase (ADA) concentration: The primary antibody used was Abcam's Anti-ADAT2 antibody (Abcam, ab122280). This antibody is homologous with the C. elegans ADA protein ADR-1 which is known to code for ADA in worms (Wormbase). The assay resulted in multiple binding sites, with distinct bands at $\sim 100 \mathrm{kd}, \sim 60 \mathrm{kd}$, and $\sim 22 \mathrm{kd}$ (Supplemental Figure S3). Samples of each band were extracted from the gel and analyzed using protein mass spectroscopy as for ADK. The band at $\sim 100 \mathrm{kd}$ contained the worm ADA homolog ADR-1, isoform D (101.8kd). 202 of the 246 samples contained ADA in sufficient concentration to be quantified by Western blotting.

\section{Metabolomics:}

To assess the relationship between enzyme concentration and activity and the concentration of their associated metabolites, we targeted four metabolites in the adenosine metabolic 
pathway: adenosine, inosine, AMP, and adenine. Several other metabolites not in the adenosine pathway were also measured, including GMP, guanine, guanosine, hypoxanthine, xanthine, and uric acid because they were part of a routine panel that included the metabolites of interest. Metabolite quantification was performed using liquid chromatography/mass spectroscopy (LC-MS), calibrated with known standards at the Southeast Center for Integrated Metabolomics at UF.

Internal standards were prepared as follows: Adenine- ${ }^{15} \mathrm{~N}_{2}$ (Cat \#A2880477), guanine-4,5- ${ }^{13} \mathrm{C}_{2} 7-{ }^{15} \mathrm{~N}$ (Cat \#G836003), hypoxanthine- ${ }^{13} \mathrm{C}_{2}{ }^{15} \mathrm{~N}$ (Cat \#H998504) and xanthine $-{ }^{13} \mathrm{C}^{15} \mathrm{~N}_{2}$ (Cat \#X499954) were purchased from Toronto Research Chemicals (Toronto, ON). Adenosine- ${ }^{15} \mathrm{~N}_{5} 5^{\prime}$-monophosphate (Cat \#662658), adenosine- ${ }^{15} \mathrm{~N}_{5} 5^{\prime}$ triphosphate (Cat \#707783), guanosine- ${ }^{15} \mathrm{~N}_{5} 5^{\prime}$-monophosphate (Cat \#900380) and guanosine- ${ }^{13} \mathrm{C}_{10} 5^{\prime}$-triphosphate (Cat \#710687) were purchased from Sigma-Adrich (St. Louis, MO). The labeled adenosine and guanosine triphosphates were dephosphorylated with alkaline phosphatase (Promega, Madison, WI; Cat \#M1821) according to the manufacturer's directions to produce the corresponding labelled nucleosides. Uric acid- ${ }^{13} \mathrm{C}$ ${ }^{18} \mathrm{O}$ was synthesized from urea- ${ }^{13} \mathrm{C}{ }^{18} \mathrm{O}$ (Cambridge Isotopes, Andover, MA; Cat \#COLM-4861) and 5,6-diaminouracil sulfate (Sigma-Aldrich; Cat \#D15103) according to methods of Cavalieri et al (Cavalieri et al. 1948).

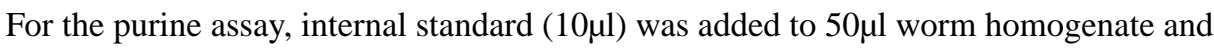
acetonitrile $(100 \mu \mathrm{l})$ was added to precipitate proteins for LC-MS/MS analysis. Samples were chromatographed on a Waters Cortecs UPLC HILIC column $(2.1 \times 150 \mathrm{~mm}, 1.6 \mu \mathrm{m})$ eluted with an acetonitrile-water gradient: Buffer A) $5 \mathrm{mmol} / \mathrm{L}$ ammonium acetate and $0.1 \%$ acetic acid in acetonitrile: water (:: 98: 2); Buffer B) $10 \mathrm{mmol} / \mathrm{L}$ ammonium formate and $0.5 \%$ formic acid in water. Mass spectrometric detection was on a Bruker EvoQ Elite MS/MS in positive ion mode, using heated electrospray ionization.

Stock solutions of the purines analyzed were prepared from authentic standards, and their concentrations determined by absorbance (Umbreit et al. 1960). The stock solutions were then mixed to give an appropriate working standard, which was then serially diluted to produce standard curves. Peak area ratios were calculated by dividing the metabolite peak area by the peak area of its isotopically labeled internal standard. Metabolite concentrations were calculated by comparing these peak area ratios to the standard curves.

\section{Data Analysis:}

(i) Estimation of mutational parameters-To quantify the cumulative effects of mutation on individual traits, we calculated the per-generation change in the average trait value ( $\Delta \mathrm{M}$, the "mutational bias") and the per-generation rate of increase in genetic variance $\left(\mathrm{V}_{\mathrm{M}}\right.$, the "mutational variance"). Mutational bias is calculated as:

$$
\Delta M=\frac{\bar{Z}_{M A}-\bar{Z}_{O}}{t \bar{Z}_{O}}
$$


where $\bar{z}_{M A}$ and $\bar{z}_{M A}$ are the means of the MA lines and the G0 pseudolines, respectively, and $t$ is the number of generations of MA ( $t=250)$ (Lynch and Walsh 1998).

The mutational variance $\left(V_{M}\right)$ is calculated as:

$$
V_{M}=\frac{V_{L, M A}-V_{L, O}}{2 t}
$$

where $V_{L, M A}$ is the among-line variance of the MA lines, $V_{L, O}$ is the among-line variance of the PS lines, and $t$ is the number of generations of MA. The among-line variance of the PS lines includes the effects of any residual segregating genetic variance, but also heritable epigenetic effects and the heritable effects of genotype-environment correlation (Lynch 1985). We report $V_{M}$ scaled by the residual variance (mutational heritability; $h_{M}^{2}=V_{M} / V_{E}$, where $V_{E}$ is the residual component of variance). The mutational covariance between traits $\left(C O V_{M}\right)$ is estimated analogously to $V_{M}$, with the among-line components of variance $\left(V_{L}\right)$ replaced with the among-line components of covariance (i.e., the off-diagonal elements in the variance-covariance matrix).

(ii) Statistical analyses-All traits except ADA and ADK concentrations and ADA activity depend on the total biomass in the sample, for which the concentration of total soluble protein is a proxy. Those three traits are expressed relative to a known amount of loaded protein and thus do not depend on the total protein in the sample. The first step in the analysis was to regress $\log$ (trait value) against $\log$ (total protein) for each trait except the three mentioned above, including the random effect of extraction block (all traits) and assay block (ADA and ADA activity); the linear model is $y_{i j}=\mu+p_{i}+b_{j}+\left(c_{k}\right)+\varepsilon_{i j}$, where $\mu$ is the overall mean, $p_{i}$ is the total protein concentration in sample $i, b_{j}$ is the random effect of extraction block $j, c_{k}$ is the random effect of assay block $k$ (for ADA and ADK activity), and $\varepsilon_{i j}$ is the residual. Residuals were checked for normality against a Q-Q plot and a few obvious outliers removed (three low, four high, $n=3514$ ). The residuals are the dependent variables in downstream analyses.

Next, we used a multivariate GLM (i.e,, MANOVA) to address the hypotheses that, averaged over all 16 traits, (i) the vector of trait means differs between the G0 ancestor and the MA lines (i.e., $|\Delta \mathrm{M}|>0$ ), and (ii) the mutational variance $\left(V_{M}\right)$ is $>0$. The full GLM can be written as: $\boldsymbol{y}_{i j k l}=\mu+a_{k}+L_{l j j}+\varepsilon_{i j k l}$, where $\boldsymbol{y}_{i j k l}$ is the vector of dependent variables measured in sample $i$ estimated as described above, $\mu$ is the overall mean, $a_{k}$ is the fixed effect of treatment (G0 or MA), $L_{l \mid j}$ is the random effect of line $I$ given treatment group $k$, and $\varepsilon_{i j k l}$ is the residual effect given treatment group $k$. This model assumes a common variance component across all traits for each random effect. Random effects (block, line, replicate) were estimated by restricted maximum likelihood (REML) with degrees of freedom determined by the Kenward-Roger method (Kenward and Roger 1997). Models were tested hierarchically, by sequential comparison of the full model, in which variance components are estimated separately for each treatment group, against a model with a single variance component for that effect, which was tested in turn against a model with the effect absent (i.e., no among-block or among-line variance). Hierarchical tests of nuisance parameters 
(block, replicate) were compared by the Bayesian Information Criterion (BIC); the model with the smallest BIC was judged the best model. The random effect of line, which is a parameter of interest, was compared by likelihood-ratio test (LRT), with degrees of freedom equal to the difference in the number of parameters estimated in the two (nested) models. The fixed effect of treatment group (G0 vs. MA) was tested by F-test on type III sums of squares. These analyses were implemented in the MIXED procedure of SAS v. 9.4.

The preceding model estimates a single variance component for each random effect, averaged across all traits, and there is no reason to expect the mutational variance will be the same for every trait. We next attempted to fit a model with the among-line variance estimated jointly for each trait (i.e., a banded main diagonal covariance structure), but the analysis failed to converge. Instead, we fit the preceding GLM for each trait $y_{i j k l}$ individually, with the relevant covariates for each trait. Statistical significance of among-line components of variance was assessed for each treatment group by LRT of models with and without the among-line component of variance constrained to zero, with the criterion of significance equal to the experiment-wide $\mathrm{a}=5 \%=0.05 / 16$.

\section{RESULTS}

\section{Per-generation change in trait means $(\Delta M)$ :}

Total soluble protein is a proxy for the total biomass in the sample. On average, after accounting for the random effects of extraction block and line, mean total protein concentration of the G0 pseudolines and the MA lines was very similar ( $2 \%$ lower in the $\mathrm{G} 0$ pseudolines; $\mathrm{F}_{1,108}=0.10, \mathrm{P}>0.75$ ). From that result we conclude that there were no systematic differences in input biomass between the two treatment groups.

Averaging over all 16 traits, MANOVA reveals a highly significant reduction in normalized trait mean in the MA lines $\left(-0.81 \times 10^{-3} /\right.$ generation; $\left.\mathrm{F}_{1,428}=24.64, \mathrm{P}<0.0001\right)$, and the point estimate of $\Delta \mathrm{M}$ is negative for every trait except ADK concentration (Table 1; Figure 4). The overall change in these metabolic traits ( -0.1\%/generation) is very similar to a wide variety of other traits quantified in these MA lines (see Supplemental Table S2 in Davies et al. 2016).

It is curious that lines with the same average biomass differ significantly in the mean of this set of metabolic traits. One possibility is that MA lines experience a general metabolic slowdown, leading to lower concentrations of these reactants, but we have no concrete evidence for that. Another possibility is that even though samples were synchronized by bleaching and were cultured to the same semi-quantitative stage of development ("a few" eggs were present on the plate), subtle differences in the distributions of developmental stages may exist at any hierarchical level in the experiment (G0 vs. MA; among lines; among replicates within a line). It is known that there are consistent changes in the genomewide transcriptional profile over the course of a few hours of development (Francesconi and Lehner 2014; Zalts and Yanai 2017), and there is reason to expect that changes in metabolite levels would change at least as fast. Determining whether a given difference in trait value between two groups is due to a true difference in the trait at the exact same stage of 
development, or due to a subtle (perhaps non-linear) difference in rate of development is a nearly insoluble problem once worms have developed past a few embryonic cell divisions.

As quantified in our enzyme activity assays, the output variable ("activity") is a function of both enzyme kinetics (i.e., activity per se) and the amount of enzyme in the sample. Interestingly, for both enzymes, the correlation of the measured activity was either negatively or not correlated with the concentration of the enzyme. In the case of ADA, the negative correlation was moderately negative and highly significant (phenotypic correlation $r$ $=-0.27, \mathrm{df}=176, \mathrm{P}<0.001)$. The correlation for ADK was near zero and not statistically significant, albeit with a smaller sample size $(r=-0.07, \mathrm{df}=113, \mathrm{P}>0.48)$. An obvious post hoc explanation is that the flux through the pathway is tightly regulated, and a change in activity per se is compensated for by a change in concentration of the enzyme in the appropriate direction, or possibly vice versa. That argument further implies, however, that the measured output of the reaction depends on factors other than the inherent activity of the enzyme itself, because at least in the PS lines the protein sequence is presumably identical in all samples (transcriptional and translational errors notwithstanding).

\section{Mutational variance (or the Lack Thereof):}

As mutations accumulate over time, MA lines are expected to diverge in trait values, leading to a consistent, long-term increase in the among-line component of variance $\left(\mathrm{V}_{\mathrm{L}}\right)$. Scaled per-generation, this increase is the "mutational variance", $\mathrm{V}_{\mathrm{M}}$ (Lynch and Walsh 1998, p. 330). For various reasons, however, some fraction of the among-line variance may be due to factors other than the accumulation of new mutations. Possible reasons include residual segregating variation in the ancestor of the MA lines, genotype-environment correlations (sometimes unknown or unknowable), and heritable epigenetic effects (Rechavi and Lev 2017; Perez and Lehner 2019). To account for potential non-genetic contributions to the among-line variance, it is necessary to include a set of "pseudolines" (PS) of the ancestor, which are treated both experimentally and statistically as if they were MA lines (Lynch 1985; Teotónio et al. 2017).

As noted in the preceding section, the mean concentration of total soluble protein did not differ between treatment groups. However, there was significant variance among MA lines after accounting for the random effect of extraction block, whereas the REML estimate of the variance among PS lines was zero, resulting in a mutational heritability $h_{M}^{2}=0.76 \times 10^{-3} /$ generation (LRT, chi-square $=4.5, \mathrm{P}<0.04$ ). The variance in total protein concentration among MA lines presumably reflects consistent differences in biomass among MA lines, which may in turn reflect differences in fecundity, growth rate, body size, or some combination thereof.

Averaging over traits and treatment groups, the multivariate GLM reveals a highly significant among-line component of variance (LRT, chi-square=135.2, $\mathrm{df}=1, \mathrm{P}<<0.0001$ ). However, a model in which the among-line variance is estimated separately for MA lines and PS lines is not significantly better than the model with only a single among-line component of variance (LRT, chi-square $=2.7, \mathrm{df}=1, \mathrm{P}>0.1$ ). When treatment groups are analyzed individually, models with an among-line component of variance are significantly 
better than models with no among-line variance for both MA lines (LRT, chi-square=113.8, $\mathrm{df}=1, \mathrm{P}<<0.0001$ ) and G0 pseudolines (LRT, chi-square $=19.3$, $\mathrm{df}=1, \mathrm{P}<0.0001$ ). Measured on the relevant scale (see Methods) and averaged over all traits, the point estimate of the among-line variance of the PS lines $(0.100+0.027)$ is about $60 \%$ of that of the MA lines $(0.166 \pm 0.021)$. Variance components and mutational heritabilities for each trait are presented in Table 2.

Conceivably, technical variance associated with enzyme or metabolite assays could swamp biological variation and lead to a spurious partitioning of variance. However, several lines of evidence suggest this is not the cause of the substantial variance among PS lines. First, and most importantly, the technical variation would have to contribute in such a way as to inflate the among-line variance of the G0 pseudolines (i.e., a rather pathological Type 1 error), rather than inflating the among-biological replicate (within-line) variance and thereby simply reducing the power to detect among-line variance. Second, we ran technical replicates (i.e., samples of extracted material were split and assayed independently) for ADK activity. The among-technical replicate component of variance was about $1 / 3$ that of the among-biological replicate variance, and pooling the technical replicates within a biological replicate or including them separately had no effect on the among-line variance. Based on previous experience with our metabolomics screen, technical replicate variance for the metabolic pools is expected to be less than $5 \%$ of the total variance for all metabolites except for GMP and uric acid, which are expected to be less than 10\% (Eoin Quinlivan, Southeast Center for Integrative Metabolomics, personal communication).

It is also very unlikely that residual segregating genetic variance could explain the similar magnitudes of the among-line variance in the PS and MA lines. First, any residual genetic variation would be equivalently partitioned among PS lines and MA lines, and would contribute equally to the among-line variance (on average, sampling variance notwithstanding). The MA lines were initiated in March, 2001, at which time the G0 ancestor was expanded to large population size (three generations) and cryopreserved. Over the intervening 16 years prior to the start of this project, the ancestor has been thawed, reexpanded, and re-frozen several times. We do not know exactly how many times the ancestor has been thawed/expanded/re-frozen, but five is a reasonable guess. If we assume that each expansion takes three generations and there have been five such expansions, then any two PS lines will have diverged for $2 \times 5 \times 3=30$ generations. In contrast, any two MA lines have diverged for $2 x(250+3) \approx 500$ generations. This is a conservative estimate because MA lines used for this experiment were also thawed and refrozen more than once, again we do not know how many times this has happened but it would inflate the number of generations of divergence between MA lines regardless of how many rounds of freeze-thaw occurred.

If technical and/or residual genetic variation cannot explain the among-line variance of PS lines, two possibilities remain. Individuals in different replicates from which data were collected ("Plate 6" in Fig. 2B) last shared a common environment four generations previous (Plate 3 in Fig. 2B) and last shared a common ancestor six generations previous (Plate 2 in Fig. 2A). The first possibility is that some feature of the environment shared by individuals on Plate 3 carried over for four generations, to be shared by all the replicates of that PS line. For example, suppose that the cryopreservation microenvironment was differently stressful 
for two PS lines (e.g., perhaps one sample sat in the glycerol freezing solution longer at room temperature before freezing than another), such that one sample ("tube 2" in Fig. 2) took longer to recover from the freeze/thaw than another. That could mean that the parents of the eggs harvested for extraction (Plate 6) were of different average ages due to their grandparents having initiated reproduction at different times and the timing differential having propagated across the intervening three generations. In that case, the proximate cause of the difference between the PS lines would be a parental effect, albeit one with origins four generations in the past.

Alternatively, it is possible that the different environmental effects shared by individuals on Plate 3 induced an epigenetic response that carried over for four generations, even though the environmental effect did not last past Plate 3. Such effects are exceptionally welldocumented in $C$. elegans and can have a variety of causes, among them parental age (Perez et al. 2017), nutritional status (Miersch and Doring 2012; Tauffenberger and Parker 2014; Jobson et al. 2015), and pathogen exposure (Rechavi et al. 2011). Recently, Sarkies and his colleagues reported that small RNA (specifically, piRNA/22G RNA) epimutations accumulate spontaneously at a rate $\sim 25 \mathrm{X}$ that of DNA sequence mutations, with a half-life on the order of 2-3 generations, but with significant fraction maintained for ten generations or more (Beltran et al. 2020). That time-scale is entirely consistent with the findings reported here.

Rechavi and Lev (2017) defined "transgenerational" epigenetic effects as those that are passed down to at least the F3 generation, in the absence of the environmental (or endogenous) trigger. It is important to note that we cannot distinguish between microenvironmental effects that are propagated across generations and thus result in conventional parental effects and "true" transgenerational epigenetic effects. In fact, unambiguously discriminating between the two possibilities in a context in which the experimenter does not control the environmental cue is extremely difficult. However, from the perspective of "things that will cause estimates of mutational variance to be misleading", the distinction is immaterial. We return to the topic of epigenetic inheritance in the Discussion.

\section{Correlations among traits}

The absence of significant mutational variance precludes estimation of mutational correlations, which was one of the underlying motivations of this study. However, because there is significant among-line variance in both the PS and MA lines, it is meaningful to investigate the among-line correlations. Note that these are not phenotypic correlations in the usual sense. Presumably, the among-line correlations reflect what might be thought of as epi-pleiotropy - the effects of an epigenetic variant (whatever it may be) on multiple traits as well as the cumulative pleiotropic effects of new mutations.

Because there are not enough lines to jointly estimate the full among-line covariance matrix, and because our primary interest is in adenosine metabolism, we restricted the analysis to the eight traits in the adenosine metabolism network. We first addressed the hypothesis that all traits are uncorrelated at the (epi)genetic level by testing the fit of a model with the full covariance matrix estimated (unrestricted covariance structure) at the level of line and 
replicate against a model with the off-diagonal elements of the among-line covariance matrix constrained to zero (banded main diagonal covariance structure), with the residual covariance matrix unconstrained. The model with the off-diagonal elements of the amongline covariance matrix constrained equal to zero provided a better fit than the model with unconstrained among-line covariances ( $\triangle \mathrm{BIC}=76.3$ units). We next tested the better model against a model with both among-line and residual covariances constrained equal to zero. The model with unstructured residual covariances fit better $(\triangle \mathrm{BIC}=15.4$ units). The average absolute within-line (residual) correlation was only about 0.12 ( $\max =0.56$; Supplemental Table S2), which shows that our total sample size $(n<290)$ provides substantial power to detect weak correlations. However, 54 lines provide little power to jointly estimate 28 among-line covariances, given that they are not predominantly large. About the most we can conclude is, first, that phenotypic correlations are weak, and second, that (epi)pleiotropy seems unlikely to be both strong and ubiquitous in the adenosine metabolism pathway.

\section{DISCUSSION}

We find ourselves confronted with an inconvenient truth: taken at face value, the results we report here are starkly contradictory to the findings reported by Davies et al. (2016). We chose the adenosine metabolism pathway for further scrutiny based on two findings of Davies et al. In that study, mean adenosine concentration increased by over $4 \%$ per generation - one of the largest values of $\Delta \mathrm{M}$ reported for any trait in any organism whereas in this study we found a decline in adenosine concentration of about $0.2 \%$ / generation in (nearly) the same set of MA lines (Table 1). Similarly, Davies et al. reported a highly significant mutational heritability $\left(\mathrm{V}_{\mathrm{M}} / \mathrm{V}_{\mathrm{E}}\right)$ for adenosine concentration of about 0.004/generation - toward the high end of mutational heritabilities (Houle et al. 1996) whereas, we found a not significant point estimate of 0.0016/generation (Table 2). The discrepancy is not restricted to a handful of traits: Davies et al. reported significant mutational variance for 22 of the 29 metabolites included in their study. Clearly, the two studies are at odds: they cant both be right, although they may both be wrong in different ways. The discrepancy is not due to the exclusion of four lines from this study, which had little effect on the results for most traits in our data (Supplemental Figure S4). The methods of quantifying metabolite concentration were different in the two studies; we used LC-MS in this study, whereas Davies et al. used GC-MS. The different platforms have different sources of variability (Gowda and Djukovic 2014), and we included total protein as a covariate whereas Davies et al. did not, either of which could in principle explain lower mutational heritabilities, although it could not explain a consistent mutational bias.

Critically, the lack of mutational variance is not because there is no variation between MA lines. For six of the 16 traits, the variance among MA lines is significantly greater than zero at the experiment-wide $5 \%$ level $(0.05 / 16 \approx \mathrm{P}<0.003)$, and four more traits are significant at the individual 5\% level (Table 2). Moreover, whether or not the among-line variance is significantly greater than zero is not random with respect to the type of trait. Of the ten metabolites, for only one (Adenine) is the among-line variance not significantly greater than zero at the individual $5 \%$ level, whereas of the six protein traits, only one (ADA concentration) has significant among-line variance at the individual 5\% level. When judged by the criteria of Davies et al., the results of the two studies are in fact quite compatible. 
Thus, the cumulative effects of mutation are not swamped by technical or microenvironmental noise (i.e., residual variance; $\mathrm{V}_{\mathrm{E}}$ ). Rather, it is that the variance among pseudolines of the ancestral control is of similar magnitude to the variance among MA lines. For economic reasons, Davies et al. did not include pseudolines of the G0 ancestor in their study. Returning to adenosine as an exemplar, all but three of the $43 \mathrm{MA}$ lines included in the Davies et al. study had mean adenosine concentrations greater than that of the G0 ancestor, which was an order of magnitude less than the mean of the MA lines in normalized units $\left(\bar{z}_{M A}=22.6 \pm 3.4, \bar{n}=3.9 ; z_{0}=2.1 \pm 0.7, n=9\right.$; see Figure 1 of Davies et al. (2016)).

Because $\Delta \mathrm{M}$ is measured relative to the ancestor, if the mean value of the ancestor is atypically small, $\Delta \mathrm{M}$ will be atypically large. We have no reason to doubt the accuracy of the estimate of mean adenosine concentration of the G0 ancestor in the Davies et al. study. 3/43 MA lines had mean concentrations lower than the ancestor, and another seven MA lines had means less than the largest of the nine replicates of the ancestor. Moreover, the average metabolite concentration of the ancestor was not low relative to the MA lines when all 29 metabolites are considered: the median rank of the ancestor is 34/44 (data from Davies et al. (2016) are archived in Dryad, at https://datadryad.org/stash/dataset/doi:10.5061/ dryad.2dn09).

It is important to carefully consider the differences between the ways the ancestral controls were treated in the two studies. At the outset of the Davies et al. study, in 2009, a single cryopreserved sample of the ancestor was thawed in the Baer lab (Florida) and plated. From that plate, a "chunk" containing hundreds of worms was transferred onto another plate and sent to the Leroi lab in England, at which time worms were washed from the plate and cryopreserved at $-80^{\circ} \mathrm{C}$. Later, one tube of the ancestor was thawed and plated onto a 100 $\mathrm{mm}$ plate. When the population on that plate reached high density (2-3 generations), worms were washed from the plate and "bleached" (Sulston and Hodgkin 1988), and surviving L1 larvae were chunked onto a new plate. From that plate, nine replicate plates were initiated from a single individual, and the populations grown to high density (2-3 generations) and synchronized by bleaching. Surviving L1s were plated and grown until worms reached young adulthood, at which time worms were collected for extraction of metabolites. In this design, the nine replicate plates are conceptually identical to the five replicates of each MA line, and the among-replicate (=within-line) variance is the residual variance, $\mathrm{V}_{\mathrm{E}}$.

In this study (depicted in Figure 3A), 15 replicate plates were initiated from a single individual, grown to high density (two generations), and cryopreserved. These are the 15 ancestral pseudolines (PS). Subsequent to thawing (depicted in Figure 3B), the PS lines were treated identically to MA lines, with five replicate plates per PS line initiated from a single individual worm taken from the thawed plate. The replicates were then propagated to the F3 descendants of the original founder of the replicate, and their offspring (F4) collected for analysis. The variation among replicates is the residual variance, $\mathrm{V}_{\mathrm{E}}$. Any effects that are common to a PS line (i.e., which contribute to $V_{L}$ ) must necessarily have been maintained at least since the replicates shared a common environment four generations previously, and potentially for as many as the six generations subsequent to the founder of the PS line.

We believe the source of the discrepancy in $\Delta \mathrm{M}$ between the two studies is likely the same as the source of the discrepancy in $\mathrm{V}_{\mathrm{M}}$ : short-term heritable (possibly epigenetic) variation. For 
example, there is a $\sim 120 \mathrm{X}$ difference between the mean adenosine concentrations between the two most extreme of the 43 MA lines in the Davies et al. study. The conventional interpretation is (and was) that spontaneous mutations accumulated over a couple of hundred generations can lead to huge differences in metabolite concentrations (and presumably in the concentrations of other biological molecules). However, there is a $\sim 100 \mathrm{X}$ difference in the mean adenosine concentration between the two most extreme of the PS lines in this study, lines that have diverged for only a few generations. If the one aliquot of the ancestor sampled in the Davies et al. study just happened by chance to fall in the lower tail of the distribution, $\Delta \mathrm{M}$ becomes "among the largest reported for any trait" (quoting Davies et al. 2016, p. 2243).

As we noted in the Results, we cannot formally distinguish between a microenvironmental effect that is propagated across generations, in which case the short-term heritability would be due to a parental effect, and a "true" transgenerational epigenetic effect. If the cause is in fact epigenetic rather than parental, what might be the cause(s), both proximate (i.e., mechanistic) and ultimate (e.g., environmental)? There is a burgeoning literature on heritable epigenetic effects in $C$. elegans, which can have a number of mechanistic causes, including several varieties of small RNA (Rechavi and Lev 2017), histone modifications (Furuhashi et al. 2010; Rechtsteiner et al. 2010; Tabuchi et al. 2018), and possibly 6-methyl adenine in DNA (Greer et al. 2015). Heritable epigenetic effects have been shown to affect a wide variety of traits (Schott et al. 2014; Demoinet et al. 2017; Han et al. 2017; Kishimoto et al. 2017), and in some cases have been shown to last for tens of generations (Ashe et al. 2012; Rechavi and Lev 2017). Parental age (Perez et al. 2017) and nutrition status (Miersch and Doring 2012; Tauffenberger and Parker 2014; Jobson et al. 2015) are especially welldocumented drivers of epigenetic variation and are obvious potential sources of variation in the experiments reported here.

Nailing down the mechanistic cause(s) responsible for the short-term heritable effects inferred here would be both very interesting and very challenging, but it is beyond the scope of this study. The most promising avenue of investigation would seem to be an experiment in which samples consisting of a small and uniform number of individuals at carefully documented developmental stages were split for combined metabolomics/transcriptomics, with a focus on piRNA/22G RNA variation (Beltran et al. 2020). However, while we do not know the mechanistic underpinning(s) of the short-term heritable variation among the ancestral pseudolines, the fact that we detected so much variation suggests that it is an important consideration in mutation accumulation studies, and more generally, in any quantitative genetic study in which phenotypic variance is partitioned within and among genotypes. Whether the high short-term heritability applies to taxa other than worms is unknown. However, a study of DNA-methylation in a set of Arabidopsis thaliana MA lines revealed that 5-methyl-cytosine epimutations occurred at a frequency several orders of magnitude greater than base substitution mutations (Becker et al. 2011). The dominant modes of epigenetic control differ between plants and nematodes ( $C$. elegans apparently does not methylate cytosine in DNA), but the general conclusion that epimutations can introduce potentially important heritable effects in the short term is unavoidable. 
In the only study comparable to this one, Clark et al. (1995, Table 3) found significant mutational heritability for the activity of 8/12 metabolic enzymes in a set of $\sim 50$ Drosophila melanogaster MA lines that had evolved under MA conditions for 44 generations. However, their assay conflates variation in enzyme activity per se and variation in enzyme concentration into the composite category "enzyme activity" (normalized by body weight and total protein concentration), without correcting for enzyme concentration. The Drosophila melanogaster genomic mutation rate is perhaps $3 \mathrm{X}$ greater than that of $C$. elegans (Sharp and Agrawal 2012; Schrider et al. 2013), which suggests that after 44 generations of MA, a Drosophila MA line would have accumulated approximately half as many mutations as one of our $C$. elegans MA lines. Contrary to our expectation based on the preceding evidence, neither of the two metabolic enzymes we assayed (ADA and ADK) exhibited among-line variance for activity per se in either the MA lines or the PS lines. Thus, for those traits, we cannot attribute the absence of $\mathrm{V}_{\mathrm{M}}$ to the confounding effects of among-line variance in the ancestor. It is interesting that the activity of these two enzymes is similarly unperturbed by both mutation and short-term heritable factors. However, neither ADA nor ADK was included in the Clark et al. study; it is certainly possible that had those enzymes been included in that study, they would have fallen in the group of enzymes without significant $\mathrm{V}_{\mathrm{M}}$.

We conclude with two thoughts. First, and more parochially, for this set of metabolic traits (enzyme activity notwithstanding), a few generations of short-term heritable effects swamp the signal of $\sim 250$ generations of accumulated mutations. Perhaps that should not be surprising: it is simply phenotypic plasticity, albeit of a different sort than evolutionary biologists are used to thinking about (Dey et al. 2016). It does strongly suggest, however, that investigators doing MA experiments need to be especially mindful of how the ancestor is treated, or employ designs in which direct comparison to an ancestor is not needed, such as regression of the among-line variance on generations of MA over multiple assays at different time points. In fact, that was the design employed in the Clark et al. study, but they constrained the intercept to equal zero, on the assumption that the among-line variance of the ancestor was zero. But also, second, and more broadly: these findings cast the recent increase in human metabolic complex disease in a different light. Although we remain skeptical of epigenetic variation as a general cause of "missing heritability" in humans, it may be that metabolic traits are particularly susceptible to epigenetic regulation and are worthy of closer scrutiny in that regard.

\section{Supplementary Material}

Refer to Web version on PubMed Central for supplementary material.

\section{Acknowledgments}

We thank L Boardman, C. Chen, and C. Short for help troubleshooting western blots, C. Howard for figure editing, J. Dembek, S. Kowalski, S. Sawtell and M. Snyder for worm husbandry, A. Saxena and M. Wayne for helpful discussions, and J. Dudycha, the anonymous reviewers and especially H. Teotónio for their insightful comments. Support was provided by a University of Florida Genetics Institute seed grant to CFB and DAH, NIH award GM107227 to CFB and E. C. Andersen, and NSF award DEB 1639005 to DAH. OJS was partially supported by NIH training grant R25GM115298. 


\section{References}

Ashe A, Sapetschnig A, Weick EM, Mitchell J, Bagijn MP, Cording AC, Doebley AL, Goldstein LD, Lehrbach NJ, Le Pen J, Pintacuda G, Sakaguchi A, Sarkies P, Ahmed S, and Miska EA. 2012 piRNAs Can Trigger a Multigenerational Epigenetic Memory in the Germline of C. elegans. Cell 150:88-99. [PubMed: 22738725]

Baer CF, Shaw F, Steding C, Baumgartner M, Hawkins A, Houppert A, Mason N, Reed M, Simonelic K, Woodard W, and Lynch M. 2005 Comparative evolutionary genetics of spontaneous mutations affecting fitness in rhabditid nematodes. Proceedings of the National Academy of Sciences 102:5785-5790.

Becker C, Hagmann J, Muller J, Koenig D, Stegle O, Borgwardt K, and Weigel D. 2011 Spontaneous epigenetic variation in the Arabidopsis thaliana methylome. Nature 480:245-U127. [PubMed: 22057020]

Beltran T, Shahrezaei V, Katju V, and Sarkies P. 2020 Epimutations driven by small RNAs arise frequently but most have limited duration in Caenorhabditis elegans. Nature Ecology \& Evolution. Early online publication.

Boison D 2013 Adenosine kinase: Exploitation for therapeutic gain. Pharmacological Reviews 65:906943. [PubMed: 23592612]

Boyle EA, Li YI, and Pritchard JK. 2017 An Expanded View of Complex Traits: From Polygenic to Omnigenic. Cell 169:1177-1186. [PubMed: 28622505]

Cavalieri LF, Blair VE, and Brown GB. 1948 The synthesis of uric acid containing isotopic nitrogen. Journal of the American Chemical Society:1240. [PubMed: 18909198]

Clark AG, Wang L, and Hulleberg T. 1995 Spontaneous mutation rate of modifiers of metabolism in Drosophila. Genetics 139:767-779. [PubMed: 7713431]

Curley JP, Mashoodh R, and Champagne FA. 2011 Epigenetics and the origins of paternal effects. Horm Behav 59:306-314. [PubMed: 20620140]

Davies SK, Leroi A, Burt A, Bundy JG, and Baer CF. 2016 The mutational structure of metabolism in Caenorhabditis elegans. 70:2239-2246.

Demoinet E, Li S, and Roy R. 2017 AMPK blocks starvation-inducible transgenerational defects in. Proc Natl Acad Sci U S A 114:E2689-E2698. [PubMed: 28289190]

Denver DR, Wilhelm LJ, Howe DK, Gafner K, Dolan PC, and Baer CF. 2012 Variation in basesubstitution mutation in experimental and natural lineages of caenorhabditis nematodes. Genome Biology and Evolution 4:513-522. [PubMed: 22436997]

Dey S, Proulx SR, and Teotonio H. 2016 Adaptation to temporally fluctuating environments by the evolution of maternal effects. PLoS Biology 14.

Eichler EE, Flint J, Gibson G, Kong A, Leal SM, Moore JH, and Nadeau JH. 2010 Missing heritability and strategies for finding the underlying causes of complex disease. Nature Reviews Genertics 11:446-450.

Francesconi M and Lehner B. 2014 The effects of genetic variation on gene expression dynamics during development. Nature 505:208-211. [PubMed: 24270809]

Furrow RE, Christiansen FB, and Feldman MW. 2011 Environment-sensitive epigenetics and the heritability of complex diseases. Genetics 189:1377-1387. [PubMed: 21968193]

Furuhashi H, Takasaki T, Rechtsteiner A, Li T, Kimura H, Checchi PM, Strome S, and Kelly WG. 2010 Trans-generational epigenetic regulation of C. elegans primordial germ cells. Epigenetics Chromatin 3:15. [PubMed: 20704745]

Gowda GAN and Djukovic D. 2014 Overview of mass spectrometry-based metabolomics: opportunities and challenges. Methods in molecular biology (Clifton, N.J.) 1198:3-12.

Greer EL, Blanco MA, Gu L, Sendinc E, Liu J, Aristizábal-Corrales D, Hsu CH, Aravind L, He C, and Shi Y. 2015 DNA Methylation on N6-Adenine in C. elegans. Cell 161:868-878. [PubMed: 25936839]

Greer EL, Maures TJ, Ucar D, Hauswirth AG, Mancini E, Lim JP, Benayoun BA, Shi Y, and Brunet A. 2011 Transgenerational epigenetic inheritance of longevity in Caenorhabditis elegans. Nature 479:365-U204. [PubMed: 22012258] 
Han S, Schroeder EA, Silva-Garcia CG, Hebestreit K, Mair WB, and Brunet A. 2017 Monounsaturated fatty acids link H3K4me3 modifiers to C. elegans lifespan. Nature 544:185-+. [PubMed: 28379943]

Hope IA 1999 C. elegans - A Practical Approach. Oxford University Press, New York.

Houle D, Morikawa B, and Lynch M. 1996 Comparing mutational variabilities. Genetics 143:14671483. [PubMed: 8807316]

Jobson MA, Jordan JM, Sandrof MA, Hibshman JD, Lennox AL, and Baugh LR. 2015 Transgenerational Effects of Early Life Starvation on Growth, Reproduction, and Stress Resistance in Caenorhabditis elegans. Genetics 201:201-+. [PubMed: 26187123]

Johnson LM, Chandler LM, Davies SK, and Baer CF. 2018 Network Architecture and Mutational Sensitivity of the C. elegans Metabolome. Frontiers in Molecular Biosciences 5:69. [PubMed: 30109234]

Kenward MG and Roger JH. 1997 Small sample inference for fixed effects from restricted maximum likelihood. Biometrics 53:983-997. [PubMed: 9333350]

Kishimoto S, Uno M, Okabe E, Nono M, and Nishida E. 2017 Environmental stresses induce transgenerationally inheritable survival advantages via germline-to-soma communication in Caenorhabditis elegans. Nat Commun 8:14031. [PubMed: 28067237]

Luna E, Bruce TJ, Roberts MR, Flors V, and Ton J. 2012 Next-generation systemic acquired resistance. Plant Physiol 158:844-853. [PubMed: 22147520]

Lynch M 1985 Spontaneous Mutations for Life-History Characters in an Obligate Parthenogen. Evolution 39:804-818. [PubMed: 28561346]

Lynch M and Walsh B. 1998 Genetics and Analysis of Quantitative Traits. Sinauer, Sunderland, MA.

Mahajan A, Taliun D, Thurner M, Robertson NR, Torres JM, Rayner NW, Payne AJ, Steinthorsdottir V, Scott RA, Grarup N, Cook JP, Schmidt EM, Wuttke M, Sarnowski C, Magi R, Nano J, Gieger C, Trompet S, Lecoeur C, Preuss MH, Prins BP, Guo X, Bielak LF, Below JE, Bowden DW, Chambers JC, Kim YJ, Ng MCY, Petty LE, Sim X, Zhang W, Bennett AJ, Bork-Jensen J, Brummett CM, Canouil M, Ec Kardt KU, Fischer K, Kardia SLR, Kronenberg F, Lall K, Liu CT, Locke AE, Luan J, Ntalla I, Nylander V, Schonherr S, Schurmann C, Yengo L, Bottinger EP, Brandslund I, Christensen C, Dedoussis G, Florez JC, Ford I, Franco OH, Frayling TM, Giedraitis V, Hackinger S, Hattersley AT, Herder C, Ikram MA, Ingelsson M, Jorgensen ME, Jorgensen T, Kriebel J, Kuusisto J, Ligthart S, Lindgren CM, Linneberg A, Lyssenko V, Mamakou V, Meitinger T, Mohlke KL, Morris AD, Nadkarni G, Pankow JS, Peters A, Sattar N, Stancakova A, Strauch K, Taylor KD, Thorand B, Thorleifsson G, Thorsteinsdottir U, Tuomilehto J, Witte DR, Dupuis J, Peyser PA, Zeggini E, Loos RJF, Froguel P, Ingelsson E, Lind L, Groop L, Laakso M, Collins FS, Jukema JW, Palmer CNA, Grallert H, Metspalu A, Dehghan A, Kottgen A, Abecasis GR, Meigs JB, Rotter JI, Marchini J, Pedersen O, Hansen T, Langenberg C, Wareham NJ, Stefansson K, Gloyn AL, Morris AP, Boehnke M and McCarthy MI. 2018 Fine-mapping type 2 diabetes loci to single-variant resolution using high-density imputation and islet-specific epigenome maps. Nat Genet 50:1505-1513. [PubMed: 30297969]

Manolio TA, Collins FS, Cox NJ, Goldstein DB, Hindorff LA, Hunter DJ, McCarthy MI, Ramos EM, Cardon LR, Chakravarti A, Cho JH, Guttmacher AE, Kong A, Kruglyak L, Mardis E, Rotimi CN, Slatkin M, Valle D, Whittemore AS, Boehnke M, Clark AG, Eichler EE, Gibson G, Haines JL, MacKay TFC, McCarroll SA, and Visscher PM. 2009 Finding the missing heritability of complex diseases. Nature 461:747-753. [PubMed: 19812666]

Marré J, Traver EC, and Jose AM. 2016 Extracellular RNA is transported from one generation to the next in Caenorhabditis elegans. Proc Natl Acad Sci U S A 113:12496-12501. [PubMed: 27791108]

Miersch C and Doring F. 2012 Paternal dietary restriction affects progeny fat content in Caenorhabditis elegans. Iubmb Life 64:644-648. [PubMed: 22639405]

Mukai T, Harada K, and Yoshimaru H. 1984 Spontaneous mutations modifying the activity of alcohol dehydrogenase (Adh) in Drosophila melanogaster. Genetics 106:73-84. [PubMed: 6420229]

Munir J, Dorn LA, Donohue K, and Schmitt J. 2001 The effect of maternal photoperiod on seasonal dormancy in Arabidopsis thaliana (Brassicaceae). Am J Bot 88:1240-1249. [PubMed: 11454624] 
Park J and Gupta RS. 2008 Adenosine kinase and ribokinase - the RK family of proteins. Cell. Mol. Life Sci 65:2875-2896. [PubMed: 18560757]

Pembrey ME, Bygren LO, Kaati G, Edvinsson S, Northstone K, Sjöström M, Golding J, and Team AS. 2006 Sex-specific, male-line transgenerational responses in humans. Eur J Hum Genet 14:159_ 166. [PubMed: 16391557]

Perez MF, Francesconi M, Hidalgo-Carcedo C, and Lehner B. 2017 Maternal age generates phenotypic variation in Caenorhabditis elegans. Nature 552:106-+. [PubMed: 29186117]

Perez MF and Lehner B. 2019 Intergenerational and transgenerational epigenetic inheritance in animals. Nature Cell Biology 21:143-151. [PubMed: 30602724]

Rando OJ and Simmons RA. 2015 I'm eating for two: parental dietary effects on offspring metabolism. Cell 161:93-105. [PubMed: 25815988]

Rasmann S, De Vos M, Casteel CL, Tian D, Halitschke R, Sun JY, Agrawal AA, Felton GW, and Jander G. 2012 Herbivory in the previous generation primes plants for enhanced insect resistance. Plant Physiol 158:854-863. [PubMed: 22209873]

Rechavi O and Lev I. 2017 Principles of Transgenerational Small RNA Inheritance in Caenorhabditis elegans. Curr Biol 27:R720-R730. [PubMed: 28743023]

Rechavi O, Minevich G, and Hobert O. 2011 Transgenerational Inheritance of an acquired small RNAbased antiviral response in C. elegans. Cell 147:1248-1256. [PubMed: 22119442]

Rechtsteiner A, Ercan S, Takasaki T, Phippen TM, Egelhofer TA, Wang W, Kimura H, Lieb JD, and Strome S. 2010 The histone H3K36 methyltransferase MES-4 acts epigenetically to transmit the memory of germline gene expression to progeny. PLoS Genet 6:e1001091. [PubMed: 20824077]

Rhee EP, Ho JE, Chen M.-h., Shen D, Cheng S, Larson MG, Ghorbani A, Shi X, Helenius IT, Donnell CJO, Souza AL, Deik A, Pierce KA, Bullock K, Walford GA, Vasan RS, Florez JC, Clish C, Yeh JJ, Wang TJ, and Gerszten RE. 2013 Resource A Genome-wide Association Study of the Human Metabolome in a Community-Based Cohort. Cell Metabolism 18:130-143. [PubMed: 23823483]

Richard MA, Huan T, Ligthart S, Gondalia R, Jhun MA, Brody JA, Irvin MR, Marioni R, Shen J, Tsai PC, Montasser ME, Jia Y, Syme C, Salfati EL, Boerwinkle E, Guan W, Mosley TH, Bressler J, Morrison AC, Liu C, Mendelson MM, Uitterlinden AG, van Meurs JB, Heijmans BT, van Meurs J, Isaacs A, Jansen R, Franke L, Boomsma DI, Pool R, van Dongen J, Hottenga JJ, van Greevenbroek MMJ, Stehouwer CDA, van der Kallen CJH, Schalkwijk CG, Wijmenga C, Zhernakova A, Tigchelaar EF, Slagboom PE, Beekman M, Deelen J, van Heemst D, Veldink JH, van den Berg LH, van Duijn CM, Hofman A, Uitterlinden AG, Jhamai PM, Verbiest M, Suchiman HED, Verkerk M, van der Breggen R, van Rooij J, Lakenberg N, Mei H, van Iterson M, van Galen M, Bot J, van 't Hof P, Deelen P, Nooren I, Moed M, Vermaat M, Zhernakova DV, Luijk R, Bonder MJ, van Dijk F, Arindrarto W, Kielbasa SM, Swertz MA, van Zwet EW, Franco OH, Zhang G, Li Y, Stewart JD, Bis JC, Psaty BM, Chen YDI, Kardia SLR, Zhao W, Turner ST, Absher D, Aslibekyan S, Starr JM, McRae AF, Hou L, Just AC, Schwartz JD, Vokonas PS, Menni C, Spector TD, Shuldiner A, Damcott CM, Rotter JI, Palmas W, Liu Y, Paus T, Horvath S, O'Connell JR, Guo X, Pausova Z, Assimes TL, Sotoodehnia N, Smith JA, Arnett DK, Deary IJ, Baccarelli AA, Bell JT, Whitsel E, Dehghan A, Levy D and Fornage M. 2017 DNA Methylation Analysis Identifies Loci for Blood Pressure Regulation. American Journal of Human Genetics 101:888-902. [PubMed: 29198723]

Sackton TB and Hartl DL. 2016 Genotypic context and epistasis in individuals and populations. Cell 166:279-287. [PubMed: 27419868]

Saklayen MG 2018 The Global Epidemic of the Metabolic Syndrome. Current Hypertension Reports 20:12. [PubMed: 29480368]

Saxena AS, Salomon MP, Matsuba C, Yeh SD, and Baer CF. 2019 Evolution of the Mutational Process under Relaxed Selection in Caenorhabditis elegans. Molecular biology and evolution 36:239-251. [PubMed: 30445510]

Schott D, Yanai I, and Hunter CP. 2014 Natural RNA interference directs a heritable response to the environment. Sci Rep 4:7387. [PubMed: 25552271]

Schrider DR, Houle D, Lynch M, and Hahn MW. 2013 Rates and genomic consequences of spontaneous mutational events in Drosophila melanogaster. Genetics 194:937-954. [PubMed: 23733788] 
Sharp NP and Agrawal AF. 2012 Evidence for elevated mutation rates in low-quality genotypes. Proc Natl Acad Sci U S A 109:6142-6146. [PubMed: 22451943]

Shin S. y., Fauman EB, Petersen A.-k., Krumsiek J, Santos R, Huang J, Arnold M, Erte I, Forgetta V, Yang T.-p., Walter K, Menni C, Chen L, Vasquez L, Valdes AM, Hyde CL, Wang V, Ziemek D, Roberts P, Xi L, Trimmer J, Theis FJ, Overington JP, Suhre K, and Brosnan MJ. 2014 An atlas of genetic influences on human blood metabolites. 46:543-550.

Sulston J and Hodgkin J. 1988 The Nematode Caenorhabditis elegansns. Pp. 587-606. Cold Spring Harbor Labratory, Cold Springs Harbor.

Tabuchi TM, Rechtsteiner A, Jeffers TE, Egelhofer TA, Murphy CT, and Strome S. 2018 Caenorhabditis elegans sperm carry a histone-based epigenetic memory of both spermatogenesis and oogenesis. Nat Commun 9:4310. [PubMed: 30333496]

Tang L and Choe KP. 2015 Characterization of skn-1/wdr-23 phenotypes in Caenorhabditis elegans; pleiotrophy, aging, glutathione, and interactions with other longevity pathways. Mechanisms of Ageing and Development 149:88-98. [PubMed: 26056713]

Tauffenberger A and Parker JA. 2014 Heritable transmission of stress resistance by high dietary glucose in Caenorhabditis elegans. PLoS Genet 10:e1004346. [PubMed: 24785260]

Teotónio H, Estes S, Phillips PC, and Baer CF. 2017 Experimental evolution with Caenorhabditis nematodes. Genetics 206:691-716. [PubMed: 28592504]

Umbreit WW, Dawson RMC, Elliott DC, and Jones KM. 1960 Data for Biochemical Research. AIBS Bulletin.

Veenendaal MV, Painter RC, de Rooij SR, Bossuyt PM, van der Post JA, Gluckman PD, Hanson MA, and Roseboom TJ. 2013 Transgenerational effects of prenatal exposure to the 1944-45 Dutch famine. BJOG 120:548-553. [PubMed: 23346894]

Wainschtein P, Jain DP, Yengo L, Zheng Z, Cupples LA, Shadyab AH, McKnight B, Shoemaker BM, Mitchell BD, Psaty BM, Kooperberg C, Roden D, Darbar D, Arnett DK, Regan EA, Boerwinkle E, Rotter JI, Allison MA, McDonald M-LN, Chung MK, Smith NL, Ellinor PT, Vasan RS, Mathias RA, Rich SS, Heckbert SR, Redline S, Guo X, Chen YDI, Liu C-T, de Andrade M, Yanek LR, Albert CM, Hernandez RD, McGarvey ST, North KE, Lange LA, Weir BS, Laurie CC, Yang J, and Visscher PM. 2019 Recovery of trait heritability from whole genome sequence data. bioRxiv.

Zalts H and Yanai I. 2017 Developmental constraints shape the evolution of the nematode middevelopmental transition. Nature Ecology \& Evolution 1:0113.

Zuk O, Hechter E, Sunyaev SR, and Lander ES. 2012 The mystery of missing heritability: Genetic interactions create phantom heritability. Proceedings of the National Academy of Sciences 109:1193-1198. 


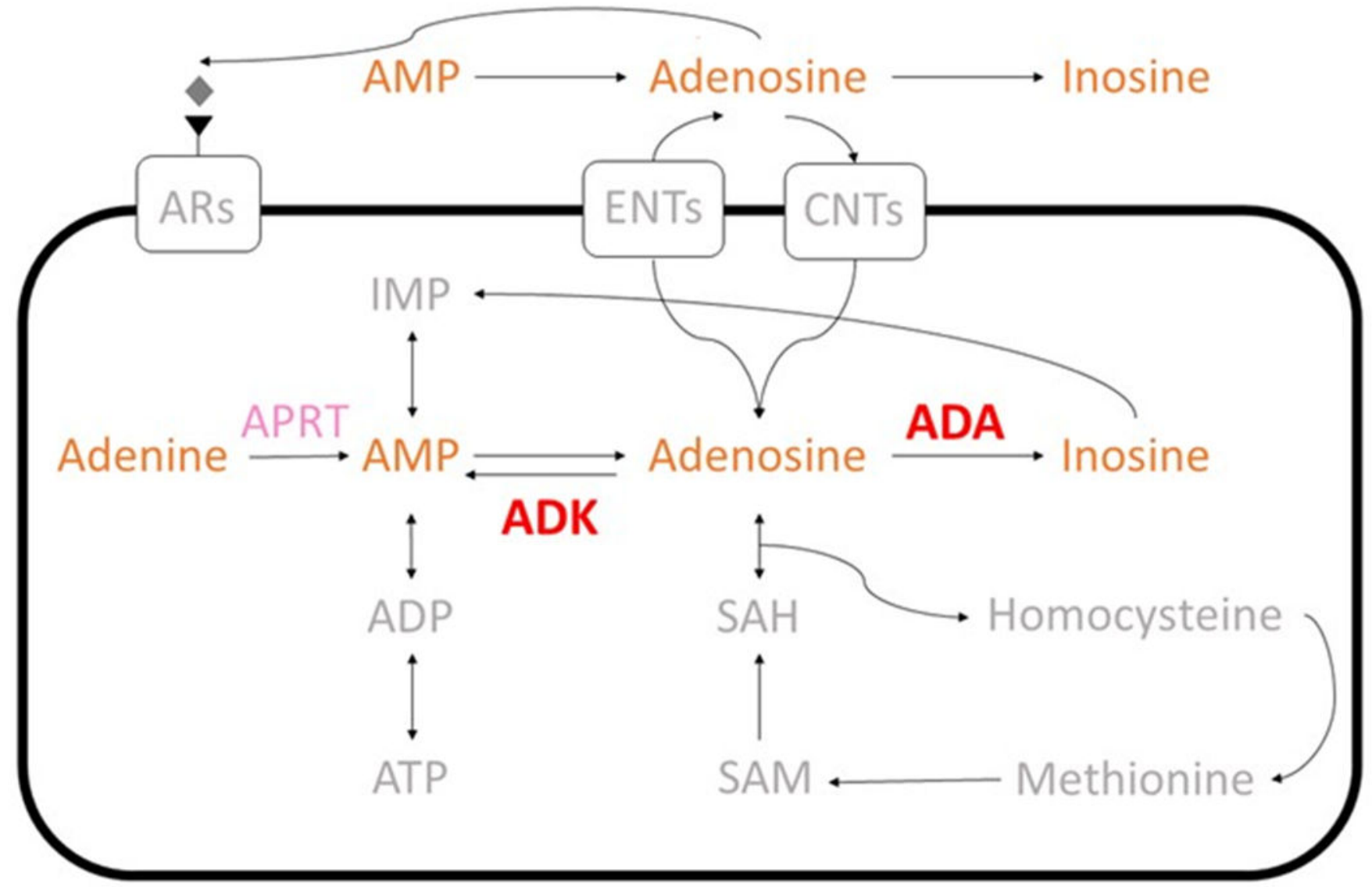

Figure 1.

Adenosine metabolism pathway. Activity and concentration of enzymes Adenosine deaminase (ADA, red) and Adenosine kinase (ADK, red) were measured. We were unable to measure the concentration of APRT (pink). Metabolites in orange had concentrations quantified, those in gray were not measured. 


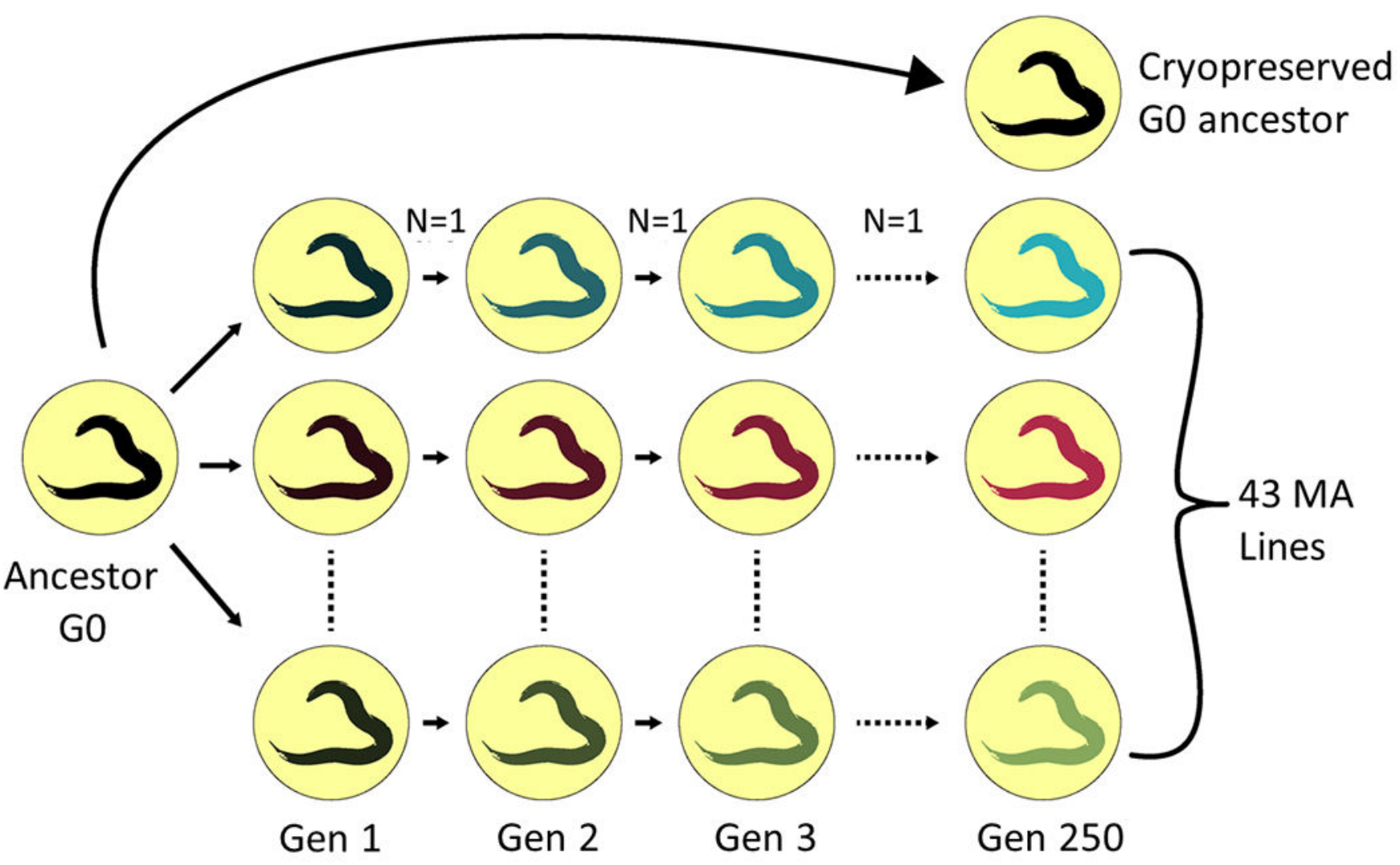

Figure 2.

Propagation of mutation accumulation (MA) lines. The G0 ancestor was thawed from a cryopreserved sample and a single hermaphrodite picked onto each of 100 agar plates. MA lines were propagated via single worm descent for $\sim 250$ generations. 43 MA lines and the G0 ancestor were included in this experiment. 
A)

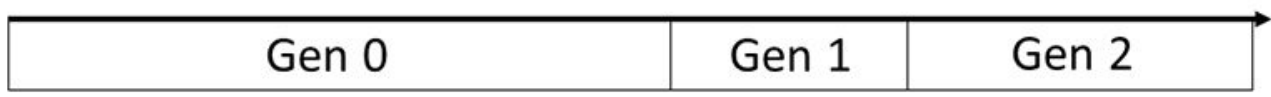

Ancestor,
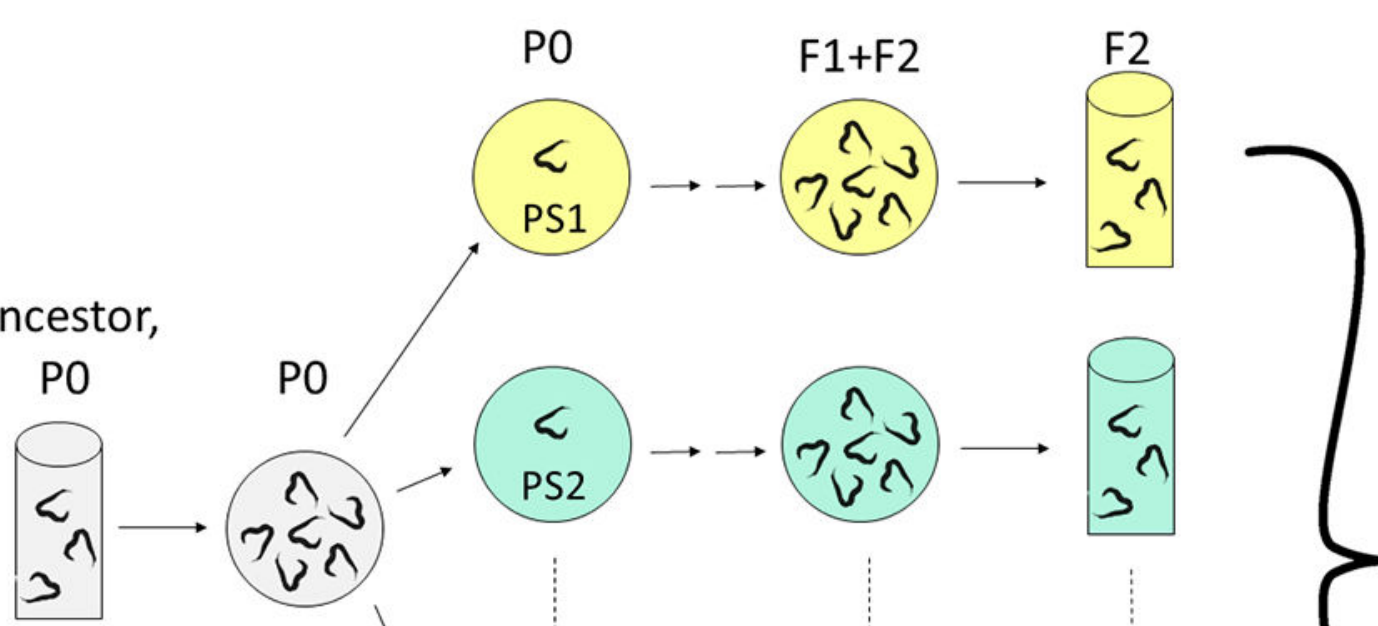

Tube 1

Plate 1
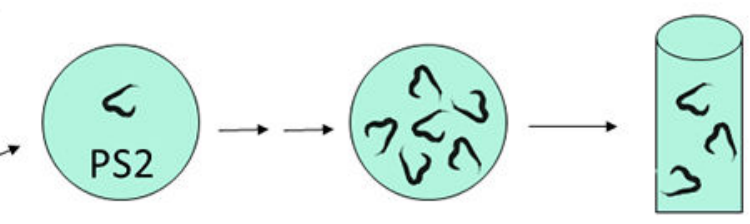


\begin{tabular}{|c|c|c|c|c|}
\hline Gen 2 & Gen 3 & Gen 4 & Gen 5 & Gen 6 \\
\hline
\end{tabular}

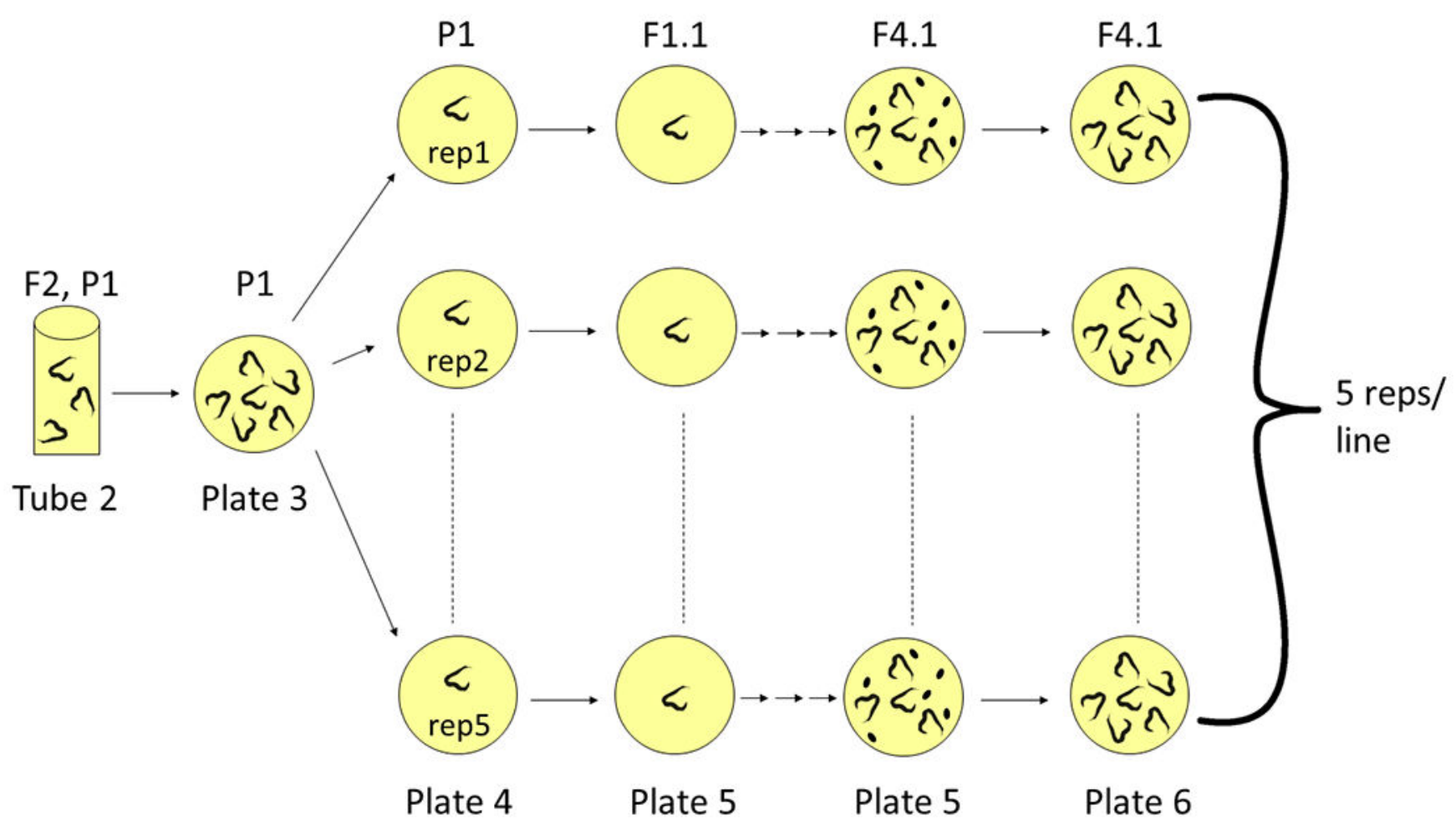

Figure 3.

A) Generation of G0 pseudolines (PS lines). The G0 ancestor was thawed from a cryopreserved sample ("Tube 1", "Plate 1") and 15 individuals were picked onto individual agar plates ("Plate 2"; PS1-PS15) and allowed to reproduce for two generations prior to cryopreservation ("Tube 2"). B) Replication of lines for protein/metabolite extraction. Lines (P1, "Tube 2" from [A]) were thawed (plate 3 ) and five individuals were picked onto individual agar plates ("Plate 4", Rep1-Rep5) and propagated by single-worm descent for another generation (F1.1, "Plate 5"). F1.1 worms were allowed to reproduce for two generations (F2.1, F3.1), and when the plates contained gravid worms (F3.1) and eggs (F4.1) they were bleached. The resulting eggs (F4.1) were transferred to a new plate ("Plate 6") and allowed to hatch and grow to the young adult stage, at which time protein and metabolites were extracted. The timeline at the top represents the number of generations of reproduction of PS lines subsequent to divergence of the lines from the common ancestor. Population sizes at each generation are summarized in tabular form in Supplemental Table S1. 


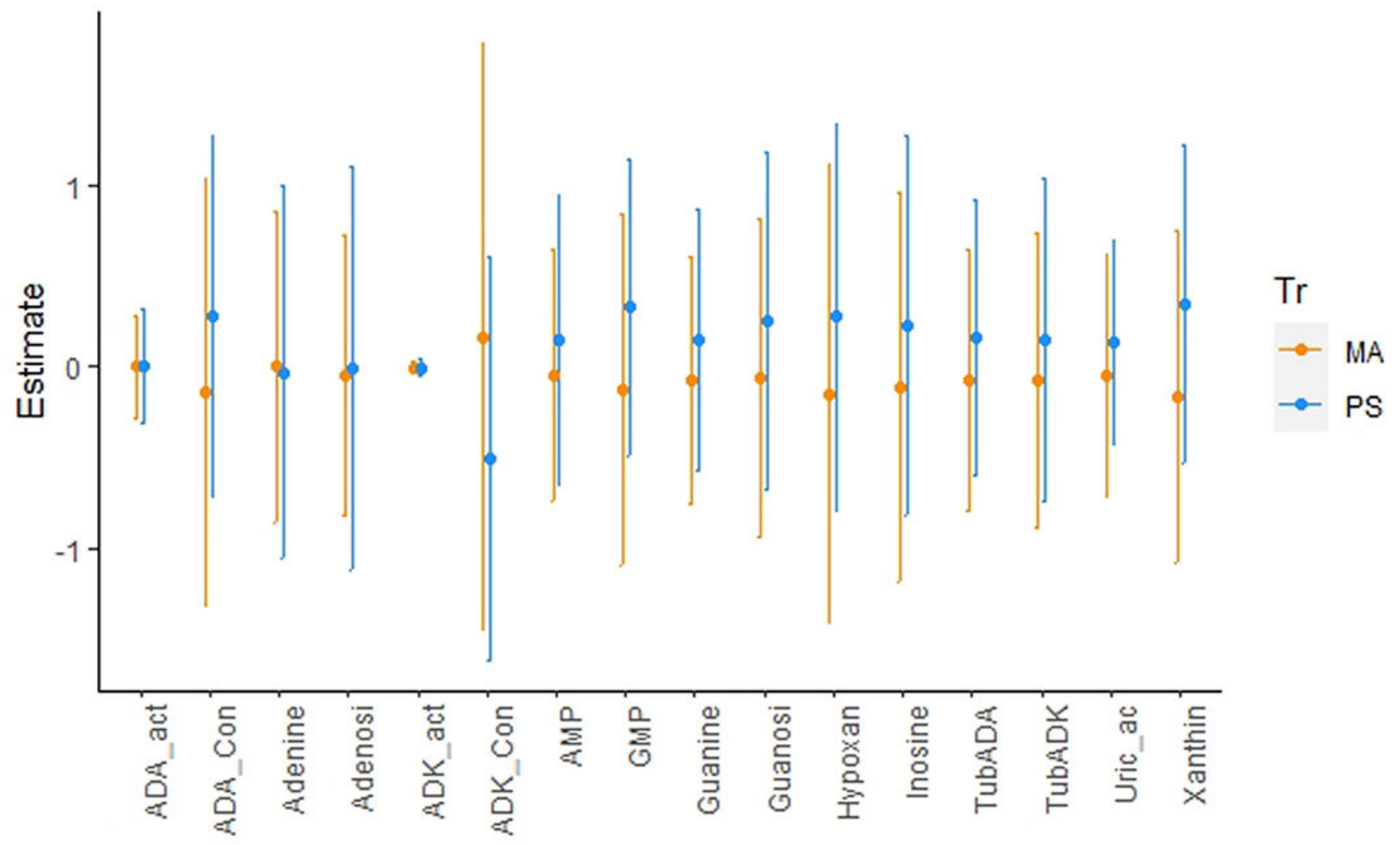

Figure 4.

Normalized trait means. Trait values are the residuals of the GLM described in the Methods. Error bars show \pm one standard deviation of line means; note that the variance in line means is distinct from the among-line component of variance, which is given in Table 2. MA lines are in orange, G0 Pseudolines in blue. 
Table 1.

Means. Column headings are: $M_{O}$, G0 pseudoline mean/median (SEM); $M_{M A}$, MA mean/median (SEM); $R_{M}$, per-generation change in trait mean conditioned on extraction block (all traits) and total protein concentration (all traits except ADA activity and ADA and ADK concentration, (SEM); $\Delta M$, per-generation change in trait mean scaled as a fraction of the G0 mean.

\begin{tabular}{|c|c|c|c|c|}
\hline Trait & $M_{G 0}$ & $M_{M A}$ & $R_{M}\left(\mathbf{x} 10^{3}\right)$ & $\Delta M\left(\mathbf{x} 10^{3}\right)$ \\
\hline ADA activity & $0.0051 / 0.0037$ (0.00042) & $0.0048 / 0.0035(0.00035)$ & $-0.15(0.40)$ & -0.24 \\
\hline ADK activity & 0.012 / $0.012(0.000128)$ & $0.012 / 0.012(0.000061)$ & $-0.001(0.0037)$ & -0.003 \\
\hline ADA conc & $0.39 / 0.29(0.10)$ & $0.25 / 0.16(0.084)$ & $-1.66(0.88)$ & -1.40 \\
\hline ADK conc & $0.62 / 0.33(0.37)$ & $3.30 / 0.71(1.11)$ & $2.67(1.16)$ & 17.18 \\
\hline Tubulin (ADA) & $0.17 / 0.17(0.02)$ & $0.13 / 0.17(0.0086)$ & $-0.95(0.56)$ & -0.81 \\
\hline Tubulin (ADK) & $0.18 / 0.18(0.021)$ & $0.13 / 0.18(0.014)$ & $-0.90(0.673)$ & -0.96 \\
\hline Total protein & $0.70 / 0.69(0.046)$ & $0.68 / 0.66(0.051)$ & $0.005(0.19)$ & -0.11 \\
\hline AMP & $11.00 / 9.75(1.62)$ & $8.53 / 7.91(1.34)$ & $-0.79(0.50)$ & -0.90 \\
\hline Adenine & $0.27 / 0.20(0.073)$ & $0.28 / 0.20(0.063)$ & $0.13(0.70)$ & -0.026 \\
\hline Adenosine & $4.67 / 0.46(2.40)$ & $2.19 / 0.35$ (1.79) & $-0.19(0.71)$ & -2.12 \\
\hline GMP & 4.26 / $3.76(0.58)$ & $2.90 / 2.16(0.43)$ & $-1.82(0.57)$ & -1.28 \\
\hline Guanine & $2.07 / 2.10(0.35)$ & $1.51 / 1.31(0.24)$ & $-0.89(0.62)$ & -1.08 \\
\hline Guanosine & 3.45 / 1.04 (1.78) & 2.08 / 0.49 (1.63) & $-1.25(0.62)$ & -1.59 \\
\hline Hypoxanthine & $5.32 / 5.20(0.91)$ & 4.19 / $3.06(0.56)$ & $-1.69(0.820)$ & -0.85 \\
\hline Inosine & $2.25 / 1.04(1.15)$ & $1.64 / 0.52(0.95)$ & $-1.38(0.85)$ & -1.09 \\
\hline Uric Acid & 16.40 / 12.05 (2.97) & $14.12 / 11.14(2.68)$ & $-0.72(0.44)$ & -0.55 \\
\hline Xanthine & $5.03 / 4.37(0.66)$ & $3.11 / 2.34(0.39)$ & $-2.06(0.64)$ & -1.53 \\
\hline Mean/Median & & & & $0.19 /-0.90$ \\
\hline
\end{tabular}




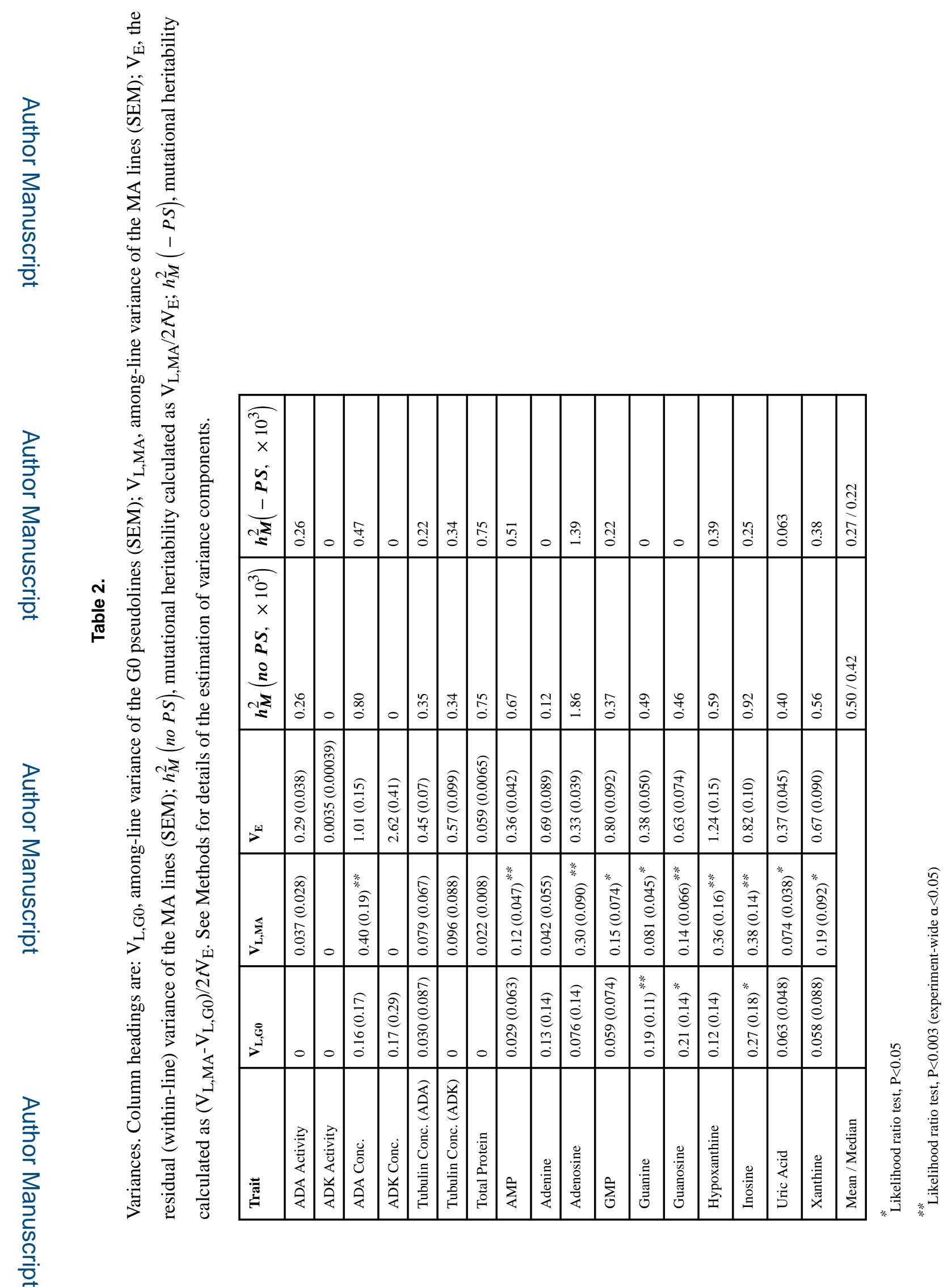

Evolution. Author manuscript; available in PMC 2021 November 01. 